\title{
Ubiquitin-specific protease 14 expression in colorectal cancer is associated with liver and lymph node metastases
}

\author{
SEIICHI SHINJI ${ }^{1,2}$, ZENYA NAITO ${ }^{2}$, SHUNJI ISHIWATA ${ }^{3}$, TOSHIYUKI ISHIWATA ${ }^{2}$, NORITAKE TANAKA ${ }^{1}$, \\ KIYONORI FURUKAWA ${ }^{1}$, HIDEYUKI SUZUKI ${ }^{1}$, TOMOKO SEYA ${ }^{1}$, AKIHISA MATSUDA ${ }^{1}$, \\ MIWAKO KATSUTA $^{1}$ and TAKASHI TAJIRI ${ }^{1}$ \\ ${ }^{1}$ Department of Surgery for Organ and Biological Regulation (Department of Surgery I), \\ Graduate School of Medicine, Nippon Medical School, 1-1-5 Sendagi, Bunkyo-ku, Tokyo 113-8603; \\ ${ }^{2}$ Department of Integrative Pathology (Department of Pathology II), Graduate School of Medicine, \\ Nippon Medical School, 1-1-5 Sendagi, Bunkyo-ku, Tokyo 113-8602; ${ }^{3}$ Faculty of Pharmaceutical Sciences, \\ Kinki University, 3-4-1 Kowakae, Higashiosaka, Osaka 577-8502, Japan
}

Received September 19, 2005; Accepted November 21, 2005

\begin{abstract}
Ubiquitin-specific protease 14, also known as the $60 \mathrm{kDa}$ subunit of tRNA-guanine transglycosylase (USP14/ TGT60kD), belongs to the ubiquitin-specific processing protease (UBP) family. USP14/TGT60kD expression in leukemic and colorectal cancer cell lines, and the suppression of such an expression after the induction of cell differentiation have been reported. In the present study, we attempted to clarify whether USP14/TGT60kD overexpression affects the clinicopathological features of colorectal cancer. Immunohistochemically, USP14/TGT60kD was absent or weakly localized in the cytoplasm of normal colorectal epithelial cells. In 18 of 99 (18.2\%) colorectal cancer patients, USP14/TGT60kD was strongly detected in the cytoplasm of cancer cells. USP14/TGT60kD expression correlated with pathological stage $(\mathrm{P}=0.03)$, and lymph node $(\mathrm{P}=0.03)$ and liver $(\mathrm{P}=0.03)$ metastases. Furthermore, the percentage of patients strongly positive for USP14/TGT60kD expression increased with pathological stage. The overall survival rate was worse in patients with a high USP14/TGT60kD expression level than in those with a low USP14/TGT60kD expression level. Our results suggest that USP14/TGT60kD also controls the fate of proteins that regulate tumor invasion and metastasis.
\end{abstract}

\section{Introduction}

Following the linking of many proteins to ubiquitin through their N-terminal amino acids, they are degraded in the 26Sproteasome in the cellular cytoplasm (1). Thus, the fate of

Correspondence to: Dr Zenya Naito, Department of Integrative Pathology, Graduate School of Medicine, Nippon Medical School, 1-1-5 Sendagi, Bunkyo-ku, Tokyo 113-8602, Japan

Email: naito@nms.ac.jp

Key words: colorectal cancer, ubiquitin-specific protease 14/USP14, tRNA-guanine transglycosylase/TGT, immunohistochemistry these proteins is determined by ubiquitination status, i.e. the balance between ubiquitination and deubiquitination. Two classes of deubiquitination enzymes have been reported $(2,3)$. One is the ubiquitin carboxy-terminal hydrolase (UCH) family, which prefers to cleave off small chains leaving groups or extended peptide chains from the carboxy-terminal of ubiquitin. Another is the ubiquitin-specific processing protease (UBP) family, which releases ubiquitin that is conjugated to various size proteins by $\alpha \mathrm{NH}$-peptide bonds and the $\varepsilon \mathrm{NH}$-isopeptide bond. Some proteins of the UBP family are associated with cancer because they control the fate of proteins that regulate cell growth and death. The herpesvirus-associated ubiquitin-specific protease (HAUSP, also known as USP7), which belongs to the UBP family, removes ubiquitin from ubiquitinated p53 and stabilizes p53, resulting in cell growth repression and apoptosis (4).

Ubiquitin-specific protease 14 (USP14) belongs to the UBP family (5). USP14 was originally cloned as a $60 \mathrm{kDa}$ subunit of tRNA-guanine transglycosylase (USP14/TGT60kD), which synthesizes queuosine, a modified nucleoside, by catalyzing the exchange reaction between the base moiety of guanosine in tRNA and a nucleobase, quanine (6). However, USP14/TGT60kD does not exhibit the exchange activity between the bases, and may be a regulation subunit of such activity. Elevated levels of queuosine were detected in leukemic cells, in which the levels of USP14/TGT60kD were also elevated (7). Our previous study on a limited number of the samples showed that the expression levels of USP14/TGT60kD are elevated in colorectal cancer tissues (8). Moreover, the expression levels of USP14/TGT60kD are decreased after the induction of cell differentiation in colorectal cancer cell lines. These findings strongly suggest a close relationship between USP14/TGT60kD expression and colorectal cancer, although the clinical significance of this relationship remains obscure.

The aim of this study is to examine USP14/TGT60kD expression in colorectal cancer patients and clarify the significance of clinicopathological features of colorectal cancer. We report that high expression levels of USP14/ 
TGT60kD were detected in 18 of $99(18.2 \%)$ colorectal cancer tissues, and correlated to the pathological stage of the cancer and lymph node and liver metastases.

\section{Materials and methods}

Patients and tissue samples. Surgical specimens were collected from 99 patients with primary colorectal adenocarcinoma and normal colonic mucosa, who underwent surgery from January to December 1998 at the Nippon Medical School Hospital (Bunkyo-ku, Tokyo, Japan). There were 64 male and 35 female patients ranging in age from 39 to 90 years (average age, 65.1 years). Table I summarizes the clinicopathological examination according to the Japanese Classification of Colorectal Carcinoma of the Japanese Society for Cancer of the Colon and Rectum (JS CCR). Patients who received preoperative chemotherapy were excluded from this study. Forty-two patients had lymph node metastasis, 14 patients had liver metastasis, 1 had lung metastasis, and 4 had peritoneal dissemination (Table I). The median follow-up period was 35.4 months. For the investigation of hematogenous metastasis and peritoneal dissemination, data on the preoperative and postoperative occurrence of metastasis were combined. This study was carried out in accordance with the principles embodied in the Declaration of Helsinki 1975.

Preparation of the antibody for USP14/TGT60kD. Recombinant USP14/TGT60kD was prepared as a fusion protein of glutathione-S-transferase (GST-USP14/TGT60kD) and a histidine tag (His-USP14/TGT60kD), as described previously (7). A Japanese white rabbit was immunized with $200 \mathrm{mg}$ of the GST-USP14/TGT60kD fusion protein mixed with Titer Max Gold (CytRx, Atlanta, GA), and serum was recovered from the animal one month after immunization. The reactivity of the anti-USP14/TGT60kD polyclonal antibody was confirmed by immunoblotting using HisUSP14/TGT60kD as the antigen, as previously described (7).

Immunohistochemistry. Paraffin-embedded sections $(3.5 \mu \mathrm{m})$ were subjected to immunostaining using the Histofine Simple Stain PO (R) Max kit (Nichirei, Tokyo, Japan). After the deparaffinization of sections in xylene and dehydration in graded alcohols, endogenous peroxidase activity was blocked by incubating the sections with $0.3 \%$ hydrogen peroxide in methanol for $30 \mathrm{~min}$. The primary antibody used in this study is a rabbit polyclonal antibody against the USP14/TGT60kD protein. The sections were then incubated with the primary antibody (1:500 dilution) in phosphate-buffered saline (PBS) containing $1 \%$ bovine serum albumin (BSA) for $16 \mathrm{~h}$ at $4^{\circ} \mathrm{C}$. Bound antibodies were detected with Simple Stain PO (R) Max reagents using diaminobenzidine-tetrahydrochloride (DAB) as the substrate, and the sections were counterstained with Mayer's hematoxylin. Negative controls were performed by omitting the primary antibodies.

Estimation of USP14/TGT60kD protein expression level. The immunoreactivity of the USP14/TGT60kD protein in colorectal cancer cells was scored based on the intensity of cytoplasmic staining using the following classification system: -, no staining; \pm , weakly and equally stained with normal mucosa;
Table I. Correlation between clinicopathological features of colorectal cancer and USP14/TGT60kD expression.

\begin{tabular}{|c|c|c|c|}
\hline \multirow[b]{2}{*}{ Variables } & \multicolumn{2}{|c|}{$\begin{array}{l}\text { USP14/TGT60kD } \\
\text { expression }\end{array}$} & \multirow[b]{2}{*}{ p-value } \\
\hline & $\begin{array}{c}\text { Low } \\
(n=81)\end{array}$ & $\begin{array}{l}\text { High } \\
(n=18)\end{array}$ & \\
\hline Sex & & & 0.59 \\
\hline Male & 51 & 13 & \\
\hline Female & 30 & 5 & \\
\hline Age & & & 0.85 \\
\hline Mean \pm 1 SD & $65.1 \pm 12.00$ & $65.7 \pm 10.64$ & \\
\hline Range & $(39-90)$ & $(49-88)$ & \\
\hline Histology & & & 0.85 \\
\hline Well & 46 & 9 & \\
\hline Moderately & 32 & 8 & \\
\hline Poorly/Muc & 3 & 1 & \\
\hline Location & & & 0.11 \\
\hline Colon & 54 & 8 & \\
\hline Rectum & 27 & 10 & \\
\hline Depth of tumor invasion & & & 0.45 \\
\hline m-sm & 13 & 1 & \\
\hline mp-si & 68 & 17 & \\
\hline p-stage & & & $0.03^{\mathrm{a}}$ \\
\hline 0 -II & 47 & 5 & \\
\hline IIIa-IV & 34 & 13 & \\
\hline Lymphatic invasion & & & 0.55 \\
\hline Positive & 61 & 15 & \\
\hline Negative & 20 & 3 & \\
\hline Vascular invasion & & & 0.18 \\
\hline Positive & 47 & 14 & \\
\hline Negative & 34 & 4 & \\
\hline Lymph node metastasis & & & $0.03^{\mathrm{a}}$ \\
\hline Positive & 30 & 12 & \\
\hline Negative & 51 & 6 & \\
\hline Liver metastasis & & & $0.02^{\mathrm{a}}$ \\
\hline Positive & 8 & 6 & \\
\hline Negative & 73 & 12 & \\
\hline Lung metastasis & & & 0.18 \\
\hline Positive & 0 & 1 & \\
\hline Negative & 81 & 17 & \\
\hline Peritoneal dissemination & & & 0.56 \\
\hline Positive & 3 & 1 & \\
\hline Negative & 78 & 17 & \\
\hline
\end{tabular}

aStatistically significant $(\mathrm{P}<0.05)$. 


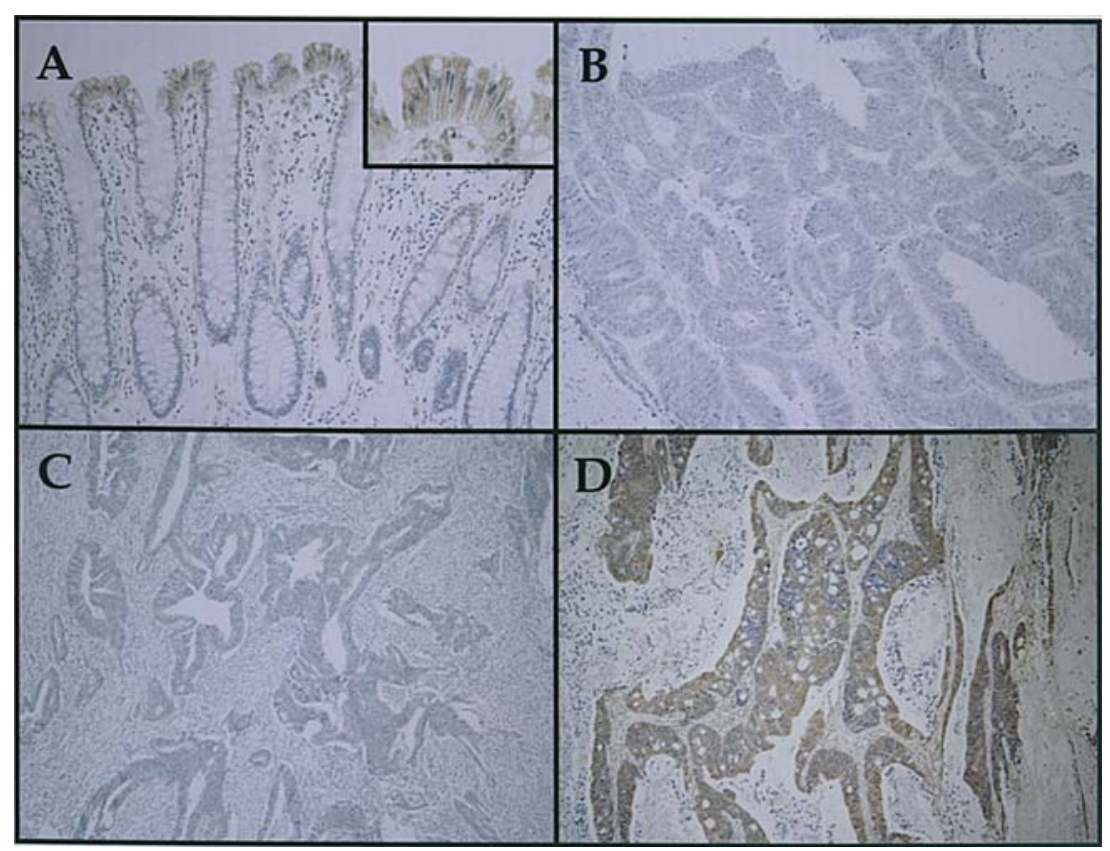

Figure 1. Representative photomicrograph of immunohistochemically stained USP14/TGT60kD. The USP14/TGT60kD protein was faintly localized in normal colorectal epithelial cells (A and inset). USP14/TGT60kD was not detected in 56 colorectal cancer patients classified into the - group (B). USP14/TGT60kD was detected more weakly than or equal to normal epithelial cells in 28 cancer patients classified into the \pm group (C). USP14/TGT60kD was strongly detected compared with normal epithelial cells in 21 patients classified into the + group (D). (Original magnification: A-D, $\mathrm{x} 200$; inset, $\mathrm{x} 400$ ).

Table II. USP14/TGT60kD expression in colorectal cancer by tumor stage.

\begin{tabular}{|c|c|c|c|}
\hline & \multicolumn{2}{|c|}{ USP14/TGT60kD expression } & \multirow{2}{*}{$\begin{array}{c}\% \text { of high } \\
\text { expression } \\
\text { cases }\end{array}$} \\
\hline & Low & High & \\
\hline Stage 0 & 8 & 0 & 0.0 \\
\hline Stage I & 12 & 1 & 7.7 \\
\hline Stage II & 27 & 4 & 12.9 \\
\hline Stage IIIa & 13 & 3 & 18.8 \\
\hline Stage IIIb & 8 & 3 & 27.3 \\
\hline Stage IV & 13 & 7 & 35.0 \\
\hline Total & 81 & 18 & 18.2 \\
\hline
\end{tabular}

and + , more strongly stained than normal mucosa. Patients positive for the USP14/TGT60kD protein were classified into the high expression cancer group, while the remaining patients were classified into the low expression cancer group. All specimens were evaluated by two investigators (S.S. and T.I) who were blinded to the clinical information of patients.

Statistical analysis. The ages of patients were compared using the Student's t-test. Correlations between USP14/TGT60kD expression and the clinicopathological features of colorectal cancer were evaluated using the Fisher's exact and Chi-square tests. The cumulative survival rate was calculated by the Kaplan-Meier method, and the significance of differences in the survival rate was analyzed by the log-rank test. $\mathrm{P}<0.05$ was considered significant in all analyses. Computations were performed using Statview J version 4.5 software (SAS Institute, Inc., Cary, NC, USA).

\section{Results}

USP14/TGT60kD expression in human colorectal cancer. Immunohistochemically, USP14/TGT60kD showed weak cytoplasmic staining in normal colorectal epithelial cells (Fig. 1A). In 54 of 99 colorectal cancer patients, USP14/ TGT60kD was not detected, and these patients were classified into the - group (Fig. 1B). USP14/TGT60kD was weakly localized in the cytoplasm of 27 cancer patients, which were classified into the \pm group (Fig. 1C). On the other hand, USP14/TGT60kD was strongly detected in 18 cancer patients, which were classified into the + group (Fig. 1D). Thus, 18 $(18.2 \%)+$ patients were classified into the high USP14/ TGT60kD expression group and $81(81.8 \%)$ - and \pm patients into the low USP14/TGT60kD expression group.

Correlation of USP14/TGT60kD expression and clinicopathological factors. The correlations between USP14/TGT60kD expression and the clinicopathological features of colorectal cancer are summarized in Table I. There were significant correlations between USP14/TGT60kD expression level and pathological stage $(\mathrm{P}=0.03)$, lymph node metastasis $(\mathrm{P}=0.03)$, and liver metastasis $(\mathrm{P}=0.02)$. On the other hand, there were no significant differences in sex, age, location, tumor depth, lymphatic invasion, vascular invasion, lung metastasis, and peritoneal dissemination. The level of high USP14/TGT60kD expression increased with tumor stage according to the pathological stage of JS CCR: $0 \%$ of stage 0 ( 0 of 8 patients), $7.7 \%$ of stage I ( 1 of 13 patients), $12.9 \%$ of stage II (4 of 31 patients), $18.8 \%$ of stage IIIa (3 of 16 patients), $27.3 \%$ of stage IIIb (3 of 11 patients), and $35.0 \%$ of stage IV (7 of 20 patients) (Table II).

Overall survival rate is worse in patients with a high USP14/TGT60kD expression level. As positive correlations 


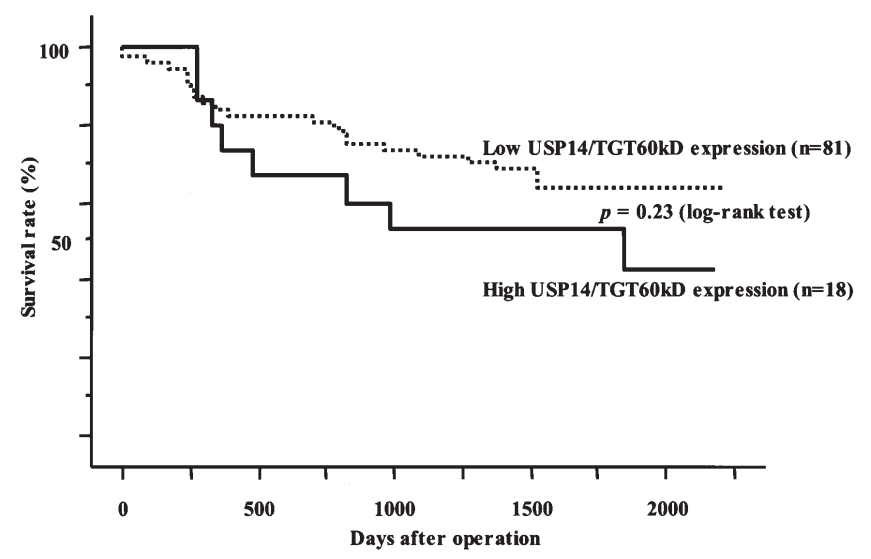

Figure 2. Kaplan-Meier overall survival curves of patients with high USP14/TGT60kD expression and low USP14/TGT60kD expression tumors. The curves represent all patients who underwent surgery $(\mathrm{n}=99)$ : 18 patients with high USP14/TGT60kD expression tumors (a) and 81 patients with low USP14/TGT60kD expression tumors (b). $\mathrm{P}=0.23$ by the log-rank test.

of USP14/TGT60kD overexpression with tumor stage, lymph node metastasis and liver metastasis, we examined the effect of USP14/TGT60kD expression on the survival of colorectal cancer patients. Kaplan-Meier survival curves show that the 5-year-survival rates were lower in the high USP14/TGT60kD expression group $(53.3 \%)$ than in the low USP14/TGT60kD expression group (64.1\%; Fig. 2). There was no significant correlation between patient survival and USP14/TGT60kD expression ( $\mathrm{P}=0.23$, log-rank test $)$.

\section{Discussion}

Previous reports have suggested the clinical significance of deubiquitinating enzymes in malignant tumors. tre- 2 is a UBP family protein, and its transcripts were detected in various malignant tumors such as bladder carcinoma and osteosarcoma, but not in nontransformed cells (9). The gene product PGP9.5 is a member of the UCH family enzymes, and elevated expression levels of PGP9.5 were observed in thyroid, lung, and pancreatic cancer as well as neuroblastoma (10-13). PGP9.5 is highly expressed in large size tumors of non-small cell lung cancer (11). A negative relationship was observed between the expression level of PGP9.5 and postoperative survival in pancreatic cancer (12). In the present study, the expression level of USP14/TGT60kD in colorectal cancer was related to the pathological stage of the cancer, and lymph node and liver metastases. These findings show the significant correlation of deubiquitinating enzymes and various types of cancer.

UBP family enzymes can prolong the lifetime of substrate proteins through deubiquitination. They possess two highly conserved domains the Cys box and His box, in their amino acid sequences $(2,3)$. These domains are related to deubiquitination activity, and other parts of the sequence contribute to the substrate specificities (14). Two substrate proteins of the UBP family have been determined to date. HAUSP/USP7 removes ubiquitin from ubiquitinated p53 and sustains the biological activities of p53 even in the presence of the ubiquitinating enzyme MDM2, resulting in cell growth repression and apoptosis (4). Another substrate is fatty acid synthase (FAS), which is a substrate of USP2a (15). USP2a removes ubiquitin from ubiquitinated FAS, and prolongs the lifetime of FAS, which results in an elevated level of FAS. Moreover, apoptosis is induced in cells with low FAS levels caused by antisense oligonucleotide transfection and siRNA experiments. The USP2a levels are elevated in prostatic cancer cell lines and cancer tissues compared with those in primary prostate tissues. Immunohistochemical analysis showed that USP2a is localized in prostate cancer tissues. The substrate proteins of the USP14 are still unclear, but these studies suggest that UBP enzymes exert their unique activities through deubiquitinating their specific substrates and sustaining the activities of these substrates.

Tumor metastasis involves a series of complex processes including the detachment of tumor cells from the primary tumor mass, microinvasion into stromal tissues, intravasation into the lymphatic or venous vessels, and extravasation and growth in secondary sites. Proteins deubiquitinated by USP14/TGT60kD in colorectal cancer are not well studied, but enhanced lymph node and liver metastases suggest that these deubiquitinated proteins are associated with both lymphatic and venous invasion pathways. Deubiquitinated proteins may play important roles in the steps common to the lymphatic and venous invasion pathways such as the detachment or microinvasion of tumor cells from primary tumors into stromal tissues. It has been shown that the invasion of cancer cells is likely to be critically regulated by the balance between activities of matrix metalloproteinases (MMPs) and their inhibitors. The secretion and activation of MMPs and naturally occurring tissue inhibitors of metalloproteinases (TIMPs) are well-regulated during normal wound healing processes, but not during invasion and metastasis (16-18). MMPs are produced by tumor cells and directly participate in human colorectal cancer invasion and metastasis (19-21). On the other hand, intercellular adhesiveness is generally reduced in human cancers. Reduced intercellular adhesiveness is also indispensable for cancer invasion and metastasis. A tumorsuppressor gene product, E-cadherin, and its undercoat proteins, catenins, which connect cadherins to actin filaments, are located at lateral borders, concentrating on the adherens junctions of epithelial cells and establish firm cell-cell adhesion (22). The balance between MMPs and TIMPs, and the E-cadherin cell adhesion system in cancer cells are inactivated by various mechanisms. Further study is needed to clarify the correlation of USP14/TGT60kD and MMPs/TIMPs or E-cadherin cell adhesion systems.

The elevated expression levels of USP14/TGT60kD were not high in the colorectal cancer patients, which suggests the applicability of a new classification system based on the levels of USP14/TGT60kD. USP14/TGT60kD-positive patients may require strict presurgical examination and follow-up programs due to frequent metastases through lymphatic and venous invasion. To our knowledge, this is the first report to show the relationship between USP14/TGT60kD expression and the clinicopathological features of colorectal cancer.

In summary, USP14/TGT60kD expression was found to be related to pathological stage, and lymphatic and liver metastases in colorectal cancer cases. This finding suggests 
the association between USP14/TGT60kD expression and metastasis in colorectal cancer.

\section{Acknowledgements}

We express our appreciation to Ms. Taeko Suzuki, Ms. Yoko Kawamoto and Ms. Kiyoko Kawahara (Department of Pathology II, Nippon Medical School) for their excellent technical assistance.

\section{References}

1. Spataro V, Norbury C and Harris AL: The ubiquitin-proteasome pathway in cancer. Br J Cancer 77: 448-455, 1998.

2. Chung $\mathrm{CH}$ and Baek SH: Deubiquitinating enzymes: their diversity and emerging roles. Biochem Biophys Res Commun 266: 633-640, 1999.

3. Wilkinson KD: Regulation of ubiquitin-dependent processes by deubiquitinating enzymes. FASEB J 11: 1245-1256, 1997.

4. Li M, Chen D, Shiloh A, et al: Deubiquitination of p53 by HAUSP is an important pathway for p53 stabilization. Nature 416: 648-653, 2002.

5. Baker RT, Wang XW, Woollatt E, White JA and Sutherland GR: Identification, functional characterization, and chromosomal localization of USP15, a novel human ubiquitin-specific protease related to the UNP oncoprotein, and a systematic nomenclature for human ubiquitin-specific proteases. Genomics 59: 264-274, 1999.

6. Deshpande KL, Seubert PH, Tillman DM, Farkas WR and Katze JR: Cloning and characterization of cDNA encoding the rabbit tRNA-guanine transglycosylase 60-kilodalton subunit. Arch Biochem Biophys 326: 1-7, 1996

7. Ishiwata S, Katayama J, Shindo H, Ozawa Y, Itoh K and Mizugaki M: Increased expression of queuosine synthesizing enzyme, tRNA-guanine transglycosylase, and queuosine levels in tRNA of leukemic cells. J Biochem 129: 13-17, 2001.

8. Ishiwata S, Ozawa Y, Katayama J, et al: Elevated expression level of $60-\mathrm{kDa}$ subunit of tRNA-guanine transglycosylase in colon cancer. Cancer Lett 212: 113-119, 2004.
9. Nakamura T, Hillova J, Mariage-Samson R, et al: A novel transcriptional unit of the tre oncogene widely expressed in human cancer cells. Oncogene 7: 733-741, 1992.

10. Takano T, Miyauchi A, Matsuzuka F, et al: PGP9.5 mRNA could contribute to the molecular-based diagnosis of medullary thyroid carcinoma. Eur J Cancer 40: 614-618, 2004.

11. Sasaki H, Yukiue H, Moriyama S, et al: Expression of the protein gene product 9.5 , PGP9.5, is correlated with T-status in non-small cell lung cancer. Jpn J Clin Oncol 31: 532-535, 2001.

12. Tezel E, Hibi K, Nagasaka T and Nakao A: PGP9.5 as a prognostic factor in pancreatic cancer. Clin Cancer Res 6: 4764-4767, 2000.

13. Yanagisawa TY, Sasahara Y, Fujie H, et al: Detection of the PGP9.5 and tyrosine hydroxylase mRNAs for minimal residual neuroblastoma cells in bone marrow and peripheral blood. Tohoku J Exp Med 184: 229-240, 1998.

14. Yin L, Krantz B, Russell NS, Deshpande S and Wilkinson KD: Nonhydrolyzable diubiquitin analogues are inhibitors of ubiquitin conjugation and deconjugation. Biochemistry 39: 10001-10010, 2000 .

15. Graner E, Tang D, Rossi S, et al: The isopeptidase USP2a regulates the stability of fatty acid synthase in prostate cancer. Cancer Cell 5: 253-261, 2004.

16. Nakajima M and Chop AM: Tumor invasion and extracellular matrix degradative enzymes: regulation of activity by organ factors. Semin Cancer Biol 2: 115-127, 1991.

17. Nagase $\mathrm{H}$ and Woessner JF Jr: Matrix metalloproteinases. J Biol Chem 274: 21491-21494, 1999.

18. Stetler-Stevenson WG, Liotta LA and Kleiner DE Jr: Extracellular matrix 6: role of matrix metalloproteinases in tumor invasion and metastasis. FASEB J 7: 1434-1441, 1993.

19. Adachi Y, Yamamoto H, Itoh F, Hinoda Y, Okada Y and Imai K: Contribution of matrilysin (MMP-7) to the metastatic pathway of human colorectal cancers. Gut 45: 252-258, 1999.

20. Ishikawa T, Ichikawa Y, Mitsuhashi M, et al: Matrilysin is associated with progression of colorectal tumor. Cancer Lett 107: 5-10, 1996

21. Emmert-Buck MR, Roth MJ, Zhuang Z, et al: Increased gelatinase A (MMP-2) and cathepsin B activity in invasive tumor regions of human colon cancer samples. Am J Pathol 145: 1285-1290, 1994.

22. Hirohashi S and Kanai Y: Cell adhesion system and human cancer morphogenesis. Cancer Sci 94: 575-581, 2003. 